\title{
DESAFÍOS ÉTICOS DE LOS CIBORGS \\ ETHICAL CHALLENGES OF CYBORGS
}

\author{
Rafael de Asís*
}

RESUMEN: Este trabajo trata sobre los desafíos éticos de los ciborgs y se divide en dos partes. En la primera se exponen algunos de los desafíos éticos que presenta la fusión entre los seres humanos y las máquinas, prestando especial atención a la discusión entre terapia y mejora. En la segunda parte se defiende la idea de que la referencia de toda regulación de esta problemática debe ser la teoría de los derechos humanos.

ABSTRACT: This paper addresses the ethical challenges of cyborgs and is divided into two parts. In the first, I present some of the ethical challenges presented by the fusion between humans and machines, with special attention to the discussion between therapy and enhancement. In the second part, I defend the idea that the reference of all regulation of this problem should be the theory of human rights.

PALABRAS CLAVE: ciborgs, terapia, mejora, derechos humanos.

KEYWORDS: cyborgs, therapy, enhancement, human rights.

Fecha de recepción: 14/02/2019

Fecha de aceptación: 07/03/2019

doi: https://doi.org/10.20318/universitas.2019.4834

* Catedrático de Filosofía del Derecho de la Universidad Carlos III de Madrid. E-mail: rarfid@inst.uc3m.es 


\section{1.- INTRODUCCIÓN}

Hace 20 años, Raymond Kurzweil publicaba La era de las máquinas espirituales ${ }^{1}$, en cuya última parte realizaba una serie de predicciones sobre cómo sería nuestro futuro. Así, entre otras cosas señalaba que, entre 2020 y 2030, los seres humanos se relacionarán con las máquinas de forma similar a como lo hacen ahora con el resto de seres humanos. En esa época, los ordenadores formarán parte de nuestras ropas y nuestras casas. Igualmente señalaba que antes de 2050, el cerebro humano podrá estar conectado directamente a la red a través de implantes cerebrales, lo que proporcionará funciones extraordinarias como aumentos de memoria o de inteligencia ${ }^{2}$.

Sthepen Hawking, en una entrevista a la BBC en 2014, señaló "La inteligencia artificial augura el fin de la raza humana" 3 . En febrero de 2017, Elon Musk ${ }^{4}$ aseguró en un evento en Dubái que o los humanos nos transformamos en organismos cibernéticos o nos convertiremos en simples mascotas de la inteligencia artificial. Yuval Noah Harari, conocido historiador israelí, en su libro publicado en 2017, Homo Deus. A Brief History of Tomorrow, se ha referido a una nueva especie (Homo Deus), fruto de una nueva filosofía (el Dataismo) como paso necesario a realizar por el Homo Sapiens, si no quiere desaparecer ${ }^{5}$. En julio de 2017, La Vanguardia publicaba un artículo, firmado por Cristina Sáez, titulado "La era de los ciborgs ya está aquí"6, en el que hacía referencia al documental "Cíborgs entre nosotros", dirigido por el periodista y realizador Rafel Durán. Eudald Carbonell, vicepresidente de Atapuerca, en una entrevista concedida a El Mundo, publicada el 7 de diciembre de 2018, afirmaba: "En el planeta a medio plazo habrá diversas especies: humana, transhumana, cyborgs...". El 16 de enero de 2019, en ABC Economía, aparecía la noticia: "Los métodos de pago se encaminan en Suecia hacia la «era ciborg»".

Para muchos, estas predicciones no dejan de ser ciencia ficción, pero, aunque así fuera, comparto la afirmación del escritor Neal Stephenson7, de que la ciencia ficción es útil para plantearnos escenarios y reflexionar sobre ellos.

\footnotetext{
1 Trad. de M.A. Galmarinien, Planeta, 1999.

2 Vid. también Kurzwei, R., La singularidad está cerca. Cuando los humanos transcendamos la Biología, Lola Books, Berlin 2012.

${ }^{3}$ Disponible en

https://www.bbc.com/mundo/ultimas noticias/2014/12/141202 ultnot hawking in teligencia artificial riesgo humanidad egn

${ }^{4}$ Presidente de Tesla y co-fundador de PayPal.

${ }^{5}$ Harari, Y.N., Homo Deus. A Brief History of Tomorrow, Vintage Penguin Random House, London 2017, pp. 298 y ss.

6 https://www.lavanguardia.com/ciencia/cienciacultura/20170703/423880164607/la-era-de-los-ciborgs-ya-esta-aqui.html

7 https://worldpolicy.org/2011/09/27/innovation-starvation/
} 
En este trabajo voy a referirme a algunos de los desafíos éticos que los ciborgs nos plantean y cuales deben ser los referentes desde los que debemos abordar esos desafíos éticos. Tengo que reconocer que buena parte de este escrito no van a ser otra cosa que dudas. $Y$ estas dudas me aparecen desde el principio, esto es, desde el propio concepto de ciborg.

Este término surge de la fusión de cibernética y organismo. Al parecer fue utilizado por primera vez por Manfred E. Clynes (científico y músico austriaco) y Nathan $\mathrm{S}$. Kline (científico estadounidense) para hacer referencia al ser humano mejorado. Así, normalmente se entiende por ciborg el ser humano con un dispositivo electrónico implantado permanentemente a su cuerpo.

Sin embargo, no todo el mundo utiliza ciborg en este sentido. Para algunos el dispositivo puede no ser electrónico. También hay quien se ha referido a ciborgs temporales (seres humanos con dispositivos implantados temporalmente 0 unidos a ellos temporalmente). Incluso, en el ámbito de la discapacidad (sobre el que volveré) el concepto ciborg se utiliza para referirse a la unión de la persona y su silla de ruedas o también, incluso, para dar cuenta de la asistencia personal y de los apoyos.

Últimamente el término se utiliza para hacer referencia a la suma de lo humano y la tecnología. Así, esta es la tesis de la antropóloga Amber Case (en su conferencia "Ahora todos somos ciborgs) o la que aparece reflejada en la sentencia del Tribunal Supremo de los Estados Unidos de 2014 en el caso Riley vs California (en donde se afirma que los móviles forman parte de la anatomía humana).

Seguramente este sea un concepto demasiado amplio. No obstante, aunque no puedo detenerme en este punto, esta manera de entender el concepto podría ser relevante a la hora de reconocer derechos...

En todo caso, lo anterior pone de manifiesto que no hay acuerdo en relación con el concepto de ciborg... Es algo parecido a lo que ocurre con el concepto de $\operatorname{robot}^{8}$, e incluso con el de ser humano ${ }^{9}$.

Por otro lado, conviene advertir que el fenómeno de los ciborgs no es un fenómeno nuevo. Ciertamente la historia de los ciborgs depende, de nuevo, del concepto que manejemos. Así, si

\footnotetext{
8 Me he referido a este problema en Una mirada a la robótica desde los derechos humanos, Dykinson, Madrid 2014, pp. 72 y ss.

9 Vid. sobre el concepto de ser humano y persona, De Lora, P. y Gascón, M., Bioética, Alianza Editorial, Madrid 2008, pp. 67 y ss. También, Martínez Morán, N., "La dignidad humana en las investigaciones biomédicas", en Marcos de Cano, A.M. (coord.), Bioética y derechos humanos, UNED, Madrid 2011, pp. 155 y ss; GarcíaLlerena, V., "Sistemas jurídicos y tradiciones culturales como barreras para un bioderecho común: el caso de la mejora en la línea germinal humana", en Análes de la Cátedra Francisco Suárez, 52, 2018, pp. 162 y ss.
} 
relacionamos ciborg con dispositivo electrónico, su historia comienza con la aparición de la ciencia ficción (literatura) a finales del XIX pero propiamente en el XX. Pero si en cambio no relacionamos ciborg con dispositivo electrónico, podemos remontarnos al 1.550 a.C., y en concreto a las prótesis de los egipcios.

En cualquier caso, en la actualidad, el fenómeno se ha acrecentado y es más visible. Así, por ejemplo, es conocida la Fundación Ciborg, creada por Neil Harbisson (el ciborg que vive con una antena en la cabeza para "escuchar" los colores) y Moon Ribas (la mujer ciborg que detecta terremotos al llevar un sensor sísmico en los pies) con la que se pretende dar voz a las identidades no humanas, defender la libertad de auto-diseño, el desarrollo de nuevos sentidos y órganos, y el derecho a mezclar el cuerpo con la máquina.

Chris Dancy es conocido por contar con 11 sensores implantados en su cuerpo que le permiten monitorear sus signos vitales las 24 horas del día.

Kevin Warwick (científico, ingeniero y profesor), se insertó, en 2002, un dispositivo en uno de sus brazos que se conectaba a su sistema nervioso, que le permitía comunicarse con su cerebro y con internet. Warwick consiguió controlar así una silla de ruedas eléctrica y una mano artificial ${ }^{10}$.

En los últimos años hay empresas que han desarrollado microchips para instalarlos en sus trabajadores y clientes, que les permiten abrir puertas, hacer fotocopias, acceder a archivos de información... Es el caso de Three Square Market o de Digiwell. En 2018, Ford España ha introducido en su planta de Valencia exoesqueletos para mejorar las condiciones de trabajo de sus trabajadores, reducir las lesiones y mejorar la ergonomía.

Podríamos citar también muchos ejemplos en el deporte, donde se discute y se plantean problemas de igualdad y salud, y en donde la biónica se está utilizando ampliamente. En este ámbito, en 2016 se celebraron las primeras Olimpiadas para ciborgs en Suiza, en los que participaron deportistas con implantes biónicos de 24 nacionalidades de todo el mundo.

No hay duda de que la actualidad de los ciborgs se ha acrecentado con el uso de las tecnologías emergentes: implantes neuronales, chips inteligentes, exoesqueletos, lentes de alcance telescópico, lentes inteligentes (capaces de grabar)...

Esta actualidad ha originado un debate sobre sus problemas y ventajas. Entre las ventajas aparece la lucha contra la enfermedad, la eficiencia, la mejora de las capacidades, el favorecimiento del ocio, etc... Entre los problemas se hace referencia a dilemas de índole ético, social, económico, medioambiental, jurídico... ${ }^{11}$.

10 http://www.kevinwarwick.com/i-cyborg/

${ }^{11}$ Incluso han aparecido asociaciones contra ciborgs: http://stopthecyborgs.org 
Y como no, esto ha llegado al campo de la reflexión filosófica en donde en relación con las nuevas tecnologías en general y su aplicación a los seres humanos, nos encontramos con dos posturas extremas: los bioconservadores y los transhumanistas ${ }^{12}$.

El bioconservadurismo defiende la conservación de la naturaleza y de su evolución natural, la igualdad, y el rechazo de la modificación del futuro. Así, Bill McKibben, escritor y activista ambiental, critica los programas de mejora humana afirmando que tener más no significa por fuerza ser más feliz. Las mejoras en el futuro, según esta posición, pueden destruir el significado de nuestras vidas ${ }^{13}$.

Por su parte, el transhumanismo es una filosofía que pretende emplear la tecnología para mejorar la vida de las personas y para resolver los problemas sociales contemporáneos. Para este movimiento, el fin de la humanidad es inevitable si no se aprovecha el desarrollo de la ciencia y la tecnología. Ese desarrollo sin límites permitirá crear máquinas singulares que puedan fusionarse con los seres humanos. Así, se defiende que las tecnologías de mejora humana deben ser ampliamente difundidas y las personas deben elegir cuál de estas tecnologías se aplican a sí mismos ${ }^{14}$. De alguna manera, la supervivencia de nuestro mundo depende de la mejora humana ${ }^{15}$

La reflexión ha llegado también al Derecho, aunque de manera lenta, centrándose principalmente en cuatro temas: la privacidad, el consentimiento, la seguridad y la responsabilidad. Este último es seguramente el tema más trabajado en el ámbito jurídico, a pesar de que lo que se nos demanda a la comunidad jurídica es seguridad más que protección ${ }^{16}$. Seguridad entendida no como seguridad personal o ciudadana, sino como seguridad jurídica, como saber a qué atenerse.

En cualquier caso, esos temas están presentes en la cuestión de los ciborgs. En el campo de la "responsabilidad Ciborg", el debate se singulariza porque se puede hablar de responsabilidad de la personaciborg (con un enfoque tradicional), de responsabilidad de la máquina-dispositivo (objetiva, por producto defectuoso) ${ }^{17}$ y

12 Vid. Llano Alonso, F.H., Homo excelsior. Los límites ético-jurídicos del transhumanismo, Tirant Lo Blanch, Valencia 2018. También, Quadra-Salcedo, T., "Retos, riesgos y oportunidades de la sociedad digital", en AA.VV., Sociedad Digital y Derecho, BOE, Madrid 2018, pp. 20 y ss.

13 Vid. McKibben, B., Enough: Staying Human in an Engineered Age, Henry Holt, Times Books, New York 2003.

14 Vid. Bostrom, N., "In Defense of Posthuman Dignity", en Bioethics, vol. 19, n. 3, 2005, pp. 202 y ss,

15 Vid. Persson, I. y Savulescu, J., Unfit for the future: the need for moral enhancement, Oxford University Press, 2012.

16 Vid. Rodotà, S., La vida y las reglas. Entre el derecho y el no derecho, Trotta, Madrid 2010, p. 12.

17 En España, artículos 135 a 149 del Real Decreto Legislativo 1/2007, de 16 de noviembre, por el que se aprueba el texto refundido de la Ley General para la 
responsabilidad de quien implanta la máquina (subjetiva, similar a la que se origina en el ámbito médico) ${ }^{18}$. Se trata, en todo caso, de un asunto de importancia cada vez mayor ${ }^{19}$.

Pero además la cuestión ciborg suscita otras cuestiones singulares en el campo jurídico, como la del comercio de las prótesis y/o dispositivos o el de su reutilización. Se trata de una cuestión que posee proyecciones de indudable calado filosófico pero también jurídico. Una de ellas es, de nuevo, la del propio concepto de persona y su relación con el cuerpo; otra es la propiedad y el uso del cuerpo humano y de sus partes 20 . Dejo para otro momento el tratamiento de esta interesante cuestión. Solo apuntaré que la reflexión sobre los ciborgs puede poner en duda el tratamiento actual de cuestiones relacionadas. Por ejemplo, la normativa vigente sobre donación de partes del cuerpo, que se aleja siempre de un posible uso comercial destacando su gratuidad 21 ; o las legislaciones restrictivas sobre la reutilización de implantes ${ }^{22}$.

Defensa de los Consumidores y Usuarios y otras leyes complementarias (en consonancia con la normativa de la Unión Europea). También es importante el 131 (sistema de seguro y Fondo de Garantía). Pueden ser responsables (sin saber bien como se reparte la responsabilidad entre ellos): quien fabrica el producto en su totalidad; quien fabrica un elemento que se integra en el producto o fabrica una materia prima del producto siempre y cuando tengan que ver con el daño; los proveedores en determinadas circunstancias; el perjudicado si participa en la producción del daño por efecto del producto. Producto defectuoso: cualquier bien mueble, aun cuando esté unido o incorporado a otro bien mueble o inmueble, así como el gas y la electricidad, siempre y cuando: (i) no ofrezca la seguridad que cabría legítimamente esperar, teniendo en cuenta todas las circunstancias y, especialmente, su presentación, el uso razonablemente previsible del mismo y los conocimientos científicos y técnicos existentes en el momento de su puesta en circulación; (ii) no ofrezca la seguridad normalmente ofrecida por los demás ejemplares de la misma serie.

18 También resultan aplicables los arts. 147 y 148 de la Ley General para la Defensa de los Consumidores y Usuarios.

${ }^{19} \mathrm{El} 30$ de noviembre de 2018 aparecía un artículo en el periódico digital eldiario.es firmado por Esther Samper y titulado "Las claves del escándalo de los implantes" en el que se informaba de la gran proliferación de problemas en relación con implantes (en los últimos 10 años se han documentado hasta 25.000 alertas).

20 Vid. al respecto García Manrique, R., "La propiedad sobre las partes separadas del cuerpo: un test para el enfoque propietaristas", en Revista Bioética y Derecho, 40, 2017, pp. 49 y ss.

21 Vid. por ejemplo los artículos primero y segundo de la Ley 30/1979, de 27 de octubre, sobre extracción y trasplante de órganos; el artículo 5 de la Ley 14/2006, de 26 de mayo, sobre técnicas de reproducción humana asistida; el artículo 7 del Real Decreto 1723/2012, de 28 de diciembre, por el que se regulan las actividades de obtención, utilización clínica y coordinación territorial de los órganos humanos destinados al trasplante y se establecen requisitos de calidad y seguridad; o el artículo 3 del Real Decreto-ley 9/2014, de 4 de julio, por el que se establecen las normas de calidad y seguridad para la donación, la obtención, la evaluación, el procesamiento, la preservación, el almacenamiento y la distribución de células y tejidos humanos y se aprueban las normas de coordinación y funcionamiento para su uso en humanos. O también el Convenio Europeo sobre Derechos Humanos y 


\section{2.- DESAFÍOS ÉTICOS. ¿QUÉ HACER? ¿CÓMO ABORDARLO?}

En todo caso, el fenómeno de los ciborgs plantea muchos desafíos éticos, y la necesidad de contestar a las preguntas ¿qué hacer? y ¿cómo abordarlo? En este punto hay dos planteamientos extremos irreales. Por un lado, el que defendería la prohibición de todo ciborg (que no es posible ya que implicaría rechazar un buen número de prácticas que hemos asumido desde hace mucho tiempo), y por otro el que defendería permitir todo ciborg (que es una postura que, en principio, puede parecer peligrosa).

¿Para qué y por qué queremos ciborgs? Una contestación habitual a estas preguntas consiste en aludir al beneficio para las personas, para uno mismo o para un tercero, o, incluso, al beneficio para la humanidad.

Como se habrá advertido, esta respuesta nos conecta con un problema conocido para la bioética: la contraposición entre terapia y mejora (entre medidas para superar enfermedades y medidas para mejorar a una persona) o entre medicina curativa y perfectiva ${ }^{23}$.

La preocupación del ser humano por mejorar sus cualidades no es algo novedoso y puede decirse que ha acompañado a la historia de la humanidad ${ }^{24}$. En la actualidad sabemos que existen empresas que ofrecen intervenciones sobre los hijos futuros ${ }^{25}$, pero también con los ya nacidos o con uno mismo ${ }^{26}$. Sin embargo, no tenemos claro el concepto de mejora y tampoco el de intervención terapéutica.

Como ha señalado Diego Gracia: "¿Dónde está el límite entre curación y mejora? Si, por ejemplo, alguien está bajo de humor y se le diagnostica de depresión, pueden dársele fármacos antidepresivos para elevarle el ánimo. ¿Hasta dónde o hasta cuándo? Se supone que hasta que alcance el nivel considerado normal. Pongamos que este nivel es 100 . Si al que tiene un humor de 80 se le puede elevar a 100 , ¿por qué no subir a 110 al que tiene 95, o al que está en 100 no

Biomedicina, de 1997, que en su artículo 21 señala: "El cuerpo humano y sus partes, como tales, no deberán ser objeto de lucro".

22 Vid. el Reglamento 2017/745 del Parlamento Europeo y del Consejo, de 5 de abril de 2017, sobre los productos sanitarios, por el que se modifican la Directiva 2001/83/CE, el Reglamento (CE) n. ${ }^{\circ} 178 / 2002$ y el Reglamento (CE) n. ${ }^{\circ}$ $1223 / 2009$ y por el que se derogan las Directivas 90/385/CEE y 93/42/CEE del Consejo. Diario Oficial de la Unión Europea L 117, de 5 de mayo de 2017.

${ }^{23}$ En el campo de la investigación se diferencia entre investigación terapéutica y no terapéutica. Vid. al respecto Pelayo González de la Torre, A., Bioética y experimentación con seres humanos, Comares, Granada 2001. pp. 37 y ss.

24 Vid. Bostrom, N., "Una historia del pensamiento transhumanista", en Argumentos de Razón Técnica, n. 14, 2011, pp. 157 y ss.

25 https://www.fertility-docs.com/programs-and-services/pgd-screening/chooseyour-babys-eye-color.php

${ }^{26}$ Es significativo el caso de la empresa Human Longevity Inc, fundada por Craig Venter y Peter Diamandis (pioneros en los campos de la genómica y la terapia con células madre), compañía privada con sede en San Diego, que se presenta como dedicada directamente a la mejora humana. 
puede elevársele a 120 ? Esto es cualquier cosa menos una pregunta retórica. Es bien sabido que ciertos ejecutivos, para rendir más en el trabajo, ser más empáticos y más simpáticos, toman Prozac. Otro ejemplo: ¿cuál es la estatura normal? A un bajito se le puede dar hormona de crecimiento para que crezca. ¿Por qué no dársela a uno normal para que pueda convertirse en un potencial jugador de baloncesto? Nadie ignora que hay todo un comercio clandestino de hormona del crecimiento. Y los ejemplos podían multiplicarse. Piénsese en la utilización de sustancias para aumentar el rendimiento deportivo, o la potencia sexual. ¿Qué es lo normal y qué lo anormal o patológico? ¿Qué es curar un defecto y qué mejorar una cualidad?"27

Además, una misma técnica o medicamento puede ser entendido como terapia o como mejora. Por ejemplo, un medicamento para tratar la hiperactividad infantil (terapia) puede utilizarse también para el rendimiento en un examen (mejora) ${ }^{28}$.

Se trata en todo caso de una distinción que posee importantes consecuencias ya que se utiliza para diferenciar lo que es o no es admisible. En este sentido, en noviembre de 2018, He Jiankiu, científico chino, era noticia en todo el mundo al afirmar que había modificado los genes de dos bebés. En concreto había modificado el gen CCR5, que el virus del sida utiliza como puerta para atacar el sistema inmunológico humano. La contestación de He Jiankiu a las críticas que inmediatamente se lanzaron por la realización de esa intervención no fue otra que la afirmación de que se trataba de una intervención terapeútica...

El informe "Human Enhancement Study" del Parlamento Europeo, de mayo de $2009^{29}$ define la intervención de mejora como aquella "destinada a mejorar el rendimiento humano individual provocada por el uso de tecnologías de base científica en el cuerpo humano". Sin embargo, se trata de una definición que no nos permite diferenciar entre intervención de mejora e intervención terapeútica, ni nos proporciona criterios para saber cuando son o no aceptables.

Dov Fox, profesor de la Universidad de San Diego, en un conocido trabajo, "The illiberality of liberal eugenics"30, defiende la idea de que la utilización de la biotecnología para asegurar la resistencia a enfermedades o para un funcionamiento cognitivo

27 Vid. Gracia, D., "Mejoramiento humano: ¿de qué estamos hablando?", en AA.VV., El mejoramiento humano. Avances, Investigaciones y reflexiones éticas y políticas, Comares, Granada 2015, p. 21.

28 Vid. al respecto Frankel, M.S. y Champman, A.R., Human Inheritable Genetic Modifications. Assesing Scientific, Ethical, Religious, and Policy Issues, Amerian Association for the Advancement of Science, September 2000.

29 Disponible en https://www.itas.Kit.edu/downloads/etag_coua09a.pdf, consultado el 13 de junio de 1014.

30 Fox, D., "The illiberality of Liberal Eugenics", en Ratio (new series) XX 1, March 2007, pp. 1 y ss. En sentido parecido se expresa Savulescu, J., "New Breeds og Humans: The Moral Obligation to Enchance", en Ethics, Law and Moral Philosophy of Reproductive Biomedicine, n. 1, 2005, pp. 36 y ss. 
correcto en relación con nuestros hijos, se puede justificar desde los mismos argumentos que utilizamos cuando defendemos la atención sanitaria o la educación básica. En muchos casos, cuando buscamos lo mejor para nuestros hijos, lo hacemos interviniendo de manera drástica en lo que podemos denominar como factores ambientales (esfuerzos que ayudan a desarrollar capacidades de los niños y que tienen que ver con nutrición, formación, ejercicios físicos) pero sin embargo, cuestionamos intervenciones sobre otros factores (como por ejemplo los genéticos) $)^{31}$.

¿Por qué ocurre esto? ¿Son tan distintos estos factores? Como señala Lydia Feito: "Por qué es más importante cultivar con esfuerzo nuestras capacidades intelectuales y afectivas en lugar de mejorarlas desde su sustrato material. Por qué pensamos que podríamos perder nuestra humanidad, nuestra identidad, por modificar nuestra condición biológica. Y por qué un "yo mejorado" es una pérdida de identidad, teniendo en cuenta que somos realidades dinámicas en permanente cambio, influidos por factores de muy diversa índole" 32 .

Se trata de algo parecido a lo que ocurre con las prótesis y aparatos que utilizamos para mejorar o corregir situaciones 0 deficiencias. Así por ejemplo, celebramos casos como el de Nathan Copeland, quien en 2014, con 28 años, diez años después de haberse lesionado la médula en un accidente perdiendo el sentido del tacto, volvió a sentir gracias a un brazo robótico directamente conectado a su cerebro ${ }^{33}$. O también el de Les Baugh, quien perdió sus dos brazos en un accidente en la década de 1970, y ahora tiene dos brazos protésicos controlados por la mente ${ }^{34}$. Hugh Herr, profesor de biofísica, que vive con dos piernas biónicas, es conocido por sus logros en ingeniería biomecánica y por ser el creador de BionX que se presenta como una empresa centrada en la mejora de la imperfección humana (en cierta manera se trata de un objetivo similar a Neuralink de Elon Musk). Pues bien, Hugh Herr fue premio Princesa de Asturias de Investigación Científica y Técnica en 2016...

Dos conocidos filósofos como J. Harris, director del Instituto de Ciencia, Ética e Innovación de la Universidad de Manchester, y M. Sandel, de la Universidad de Harvard, consideran que sortear la

31 Vid. al respecto Vid. Buchanan, A., Brock, D.W., Daniels, N., y Wikler, D., Genética y Justicia, trad. de C. Piña, Cambridge University Press, Madrid 2002, pp. 150 y ss.

32 Vid. Feito, L., "Neurofármacos y mejora humana", en AA.VV., El mejoramiento humano. Avances, Investigaciones y reflexiones éticas y políticas, cit., pp. 94 y ss.

33 Vid. Flesher; S.N., Collinger, J.L., Foldes, S.T., Weiss, J.M., Downey, J.E., TylerKabara, E.C., Bensmaia, S.J., Schwartz, A.B., Boninger, M.L., Gaunt, R.A., "Intracortical microstimulation of human somatosensory cortex", en Science Translational Medicine, Vol. 8, Issue 361, 2016, pp. 361 y ss.

34 Vid. Chi, A., Smith, S., Womack, I. Armiger, R., "The Evolution of Man and Machine-a Review of Current Surgical Techniques and Cutting Technologies After Upper Extremity Amputation", en Current Trauma Reports, Volume 4, Issue 4, December 2018, pp. 339 y ss. 
adversidad o negociar con lo recibido son constantes humanas, sin embargo, a partir de ahí, uno defiende la introducción sin límite de mejoras y el otro, por el contrario, las rechaza ${ }^{35}$.

Carlos Lema, en quien me voy a apoyar a la hora de analizar el concepto de mejora, en su trabajo "Intervenciones biomédicas de mejora, mejoras objetivas y mejoras discriminatorias: ¿De la Eugenia al darwinismo social?"36, ha propuesto acotar el debate en términos razonables pensando en cuando la intervención nos plantea dudas y por qué nos plantea dudas.

En este punto solemos utilizar estándares de normalidad... Así, el límite de la mejora algunos lo ponen en ir mas allá de lo normal, en alcanzar atributos o capacidades fuera de lo normal. Pero este es precisamente, como señala el propio Lema, uno de los problemas del concepto de mejora: partir de un concepto de lo normal que es confuso, y que no está claro que, de existir, posea valor moral.

Aún así, es posible diferenciar, con George Khushf (Director del Centro de Bioética de la Universidad de Carolina del Sur), entre mejoras incrementales (modestas, medibles y evaluables) y mejoras radicales (permanentes, irreversibles y no evaluables). Ejemplos de las primeras serían la cirugía estética o los fármacos para la mejora cognitiva, y ejemplos de las segundas una técnica para lograr una visión especial o para establecer nuevas formas de comunicación ${ }^{37}$.

Veamos dos ejemplos diferentes y tres posibles situaciones en cada uno de ellos.

El primer ejemplo tiene que ver con la implantación de una especie de exoesqueleto a una persona que posee una lesión en la espalda. Pues bien, supongamos que: (i) se implanta un exoesqueleto a una persona que la permite trabajar en una fábrica en las mismas condiciones que cualquier otra; (ii) se implanta un exoesqueleto a una persona que la permite trabajar en una fábrica pero en trabajos especiales que requieren condiciones físicas también especiales sólo al alcance de unos pocos; (iii) se implanta un exoesqueleto a una persona que la permite trabajar en una fábrica pero en trabajos que requieren condiciones físicas nunca conseguidas por los seres humanos.

El segundo ejemplo tiene que ver con la implantación de un chip en el oído que permite la comprensión de un idioma. Pues bien, supongamos que: (i) se implanta a una persona un chip que permite la comprensión del inglés; (ii) se implanta a una persona un chip que permite la comprensión del inglés, el francés y el español; (iii) se

\footnotetext{
35 Vid. Harris, J., Enchancing Evolution. The Ethical Case for Making Better People, Princeton University Press, Princeton 2007, p. 9; Sandel, M., Contra la perfección, Marbot, Barcelona 2007, p. 127.

${ }^{36}$ En Anales de la Cátedra Francisco Suárez, 49, 2015, pp. 367 y ss.

37 Khushf, G., "Stage Two Enhancements", en F. Jotterand (ed.), Emerging Conceptual, Ethical and Policy Issues in Bionanotechnology, Springer, 2008, pp. 203 y ss.
} 
implanta a una persona un chip que permite la comprensión de 1.000 lenguas.

El primer caso del primer ejemplo puede ser entendido como una intervención terapéutica, mientras que el resto serían intervenciones de mejora. Por su parte, terceras intervenciones podrían ser de mejora radical y el resto de mejora incremental, siguiendo la terminología de Khushf. El primero de los casos, además, introduce una variable que trataré más adelante: la discapacidad.

Pero en todo caso, desde un punto de vista ético, podríamos afirmar que sólo los casos de mejora radical nos producen complicaciones. La intervención terapeútica y las de mejora incremental se pueden asemejar a otras intervenciones que aceptamos sin problema. En cambio, las de mejora radical nos llevan a escenarios desconocidos respecto a los cuales no tenemos referentes ni criterios éticos.

Ahora bien, como señala Carlos Lema, en estos casos para los que no tenemos referentes morales inmediatos, además de la introducción de cambios inéditos en la naturaleza, nos preocupa también tener el poder de decisión de llevar a cabo este tipo de intervenciones 38 .

Ha señalado M. Sandel que el principal peligro del perfeccionamiento y la ingeniería genética radica "en que son el reflejo de una ampliación desmesurada del campo de la acción humana, de una aspiración prometeica a rehacer la naturaleza, incluida la naturaleza humana, para servir a nuestros propósitos y satisfacer nuestros deseos" 39 .

Es común en este punto hacer referencia al argumento de la pendiente resbaladiza o del plano inclinado. En virtud de este argumento, no se deberían realizar ciertas actuaciones porque aunque produzcan en un primer término buenas consecuencias, son a la vez causa de otras que determinarán a su vez otras que finalmente nos conducirán a malas consecuencias. Así, si queremos evitar ese desenlace no debemos realizar el primer paso ${ }^{40}$.

Ciertamente, para superar la preocupación de la que nos habla Lema, esa inclinación humana a la que se refiere Sandel, o el propio argumento de la pendiente resbaladiza, nos ayudará el comprobar que existen mejoras objetivas, esto es, mejoras buenas para todos. Así por ejemplo, Peter Singer distingue entre mejoras absolutas u

38 Lema, C., "Intervenciones biomédicas de mejora, mejoras objetivas y mejoras discriminatorias: ¿De la Eugenia al darwinismo social?", cit., p. 374.

${ }^{39}$ Sandel, M., Contra la perfección, cit., p. 39.

40 En un sentido crítico con este argumento vid. De Miguel Beriain, I. y Armaza Armaza, E., "Un análisis ético de las nuevas tecnologías de edición genética: el CRISOR-Cas9 a debate", en Análes de la Cátedra Francisco Suárez, 52, 2018, pp. 193 y ss. También Junquera de Estéfani, R., "El Bioderecho", en Marcos del Cano, A.M. (coord.), Bioética y derechos humanos, cit., pp. 139 y ss. 
objetivas y mejoras relativas, señalando como ejemplo de las primeras el aumento de la longevidad ${ }^{41}$.

En sentido parecido se expresa Savulescu refiriéndose a la mejor vida, entendiendo por tal aquella en la que se puede disfrutar de mayor bienestar, y en relación con la cual formula el "Principio de beneficencia procreativa" según el cual las parejas deben seleccionar entre los hijos posibles aquel que se espera vaya a tener una vida mejor. No hacerlo sería algo inmoral ${ }^{42}$.

Ahora bien, es posible cuestionar ese carácter absoluto de la longevidad 43 o el de mejor vida, y pensar, por el contrario que el concepto de mejora es relativo y puede cambiar según contextos o culturas.

En este punto podemos referirnos al exjugador de baloncesto de la NBA Yao Ming, de 2,29 metros de altura ${ }^{44}$. Claramente su altura suponía una ventaja para la práctica del baloncesto y seguramente para ganar una importante cantidad de dinero con su práctica. Una persona podría buscar alcanzar esa altura para conseguir esas ventajas. Pero está claro que se trata de una cuestión contextual. Esta altura puede ser buena para algunas cosas pero no para otras, como lo demuestra el llamado "Club de los altos y altas en Chile" (institución creada por personas altas para compartir sus problemas) ${ }^{45}$

Ahora bien, incluso aceptando que existan mejoras objetivas, Carlos Lema plantea la posibilidad de que pueden ser discriminatorias y carecer de justificación.

Nicholas Agar, autor australiano que se describe como a medio camino entre bioconservadores y transhumanistas, plantea el

41 Vid. Singer, P., "De compras por el supermercado genético", trad. de J. Seoane, Isegioria n. 27, 2002, p. 36. Otra defensa de la existencia de mejoras objetivas puede verse en Fox, D., "The illiberality of Liberal Eugenics", cit., pp. 1 y ss.

42 Vid. Savulescu, J., "Procreative Beneficence: Why We Should Select the Best

Children", en Bioethics 15, 2002, pp. 413 y ss.

43 Así por ejemplo B. Williams, considera que la vida solo tiene sentido como existencia finita. Vid. Williams, B., "The Makropulos Case: Reflections on the Tedium of Immortality", en Problems of The Self, Cambridge University Press, Cambridge 1973, p. 89.

${ }^{44}$ Carlos Lema pone como ejemplo en este punto a Sharon Duchesneau y Candace McCullough, quienes en 2002, hicieron pública su decisión de tener un hijo mediante inseminación artificial. La particularidad de su caso es que ambas eran sordas y habían decidido que sus hijos también lo fueran. Como es sabido, los miembros de la denominada como Comunidad Sorda, se consideran como una minoría cultural que comparte los rasgos típicos de cualquier otro grupo cultural, siendo el principal el de la lengua de signos. Desde la Comunidad Sorda, la persona sorda se define como aquella que usa la lengua de signos.

45 No es extraño así que haya quien defienda que estos problemas deben ser resueltos desde criterios culturales y contextuales. Por ejemplo, se afirma (Coenen, Schuiijff y Smits, "The Politics of Human Enhancement and the European Union", en Savulescu, J., Meulen, R. y Kahane, G. (eds.), Enhancing Human Capacities, WileyBlackwell, Oxford 2011) que, en el contexto de la Unión Europea, las tecnologías de mejora humana tienen que ser evaluadas desde los valores y creencias europeos. 
supuesto de una situación en la que los padres tienen la posibilidad de alterar, mediante tecnologías de modificación genética, el color de la piel de sus hijos. Es posible así tanto oscurecer el color de la piel, como medida de protección del sol ante la disminución de la capa de ozono, como aclararla, para evitar la discriminación en una sociedad racista. Agar defiende la primera de las opciones pero, sin embargo, señala que no debería permitirse la segunda. Y lo justifica apelando a la idea de discriminación y al rechazo de la idea de que el valor moral de una persona pueda depender del color de su piel ${ }^{46}$.

Como señala Carlos Lema, "en una sociedad racista, el poseer unos determinados rasgos fenotípicos asociados a una raza puede ser causa de sufrimiento, de rechazo social y de reducción de las oportunidades disponibles. Igualmente, desde esta idea de construcción social de los valores, en una sociedad sexista, por ejemplo, podría llegar a considerarse una mejora el seleccionar varones en lugar de mujeres" 47.

Volviendo a los ciborgs, imaginemos que, la pérdida de una pierna nos hace ir en una silla de ruedas y enfrentarnos a muchas barreras, pero nos encontramos con la posibilidad de colocarnos una prótesis teniendo dos opciones. Una de ellas consiste en colocar una prótesis que realiza todas las funciones de la pierna que nos falta pero no tiene aspecto de pierna; la otra es colocar una prótesis menos funcional pero que es idéntica a una pierna. Alguien podría pensar que no hay duda en la elección: hay que optar por la primera por su funcionalidad. Sin embargo, algunos tendrían dudas porque considerarían que esa prótesis extraña, trasladaría esa calificación a la persona que la lleva, por lo que sería mejor optar por la segunda. Optar por esta solución apelando a esta razón implica ser condescendiente con la situación de discriminación en la que se encuentran muchas personas con discapacidad.

En el caso de los ciborgs, puede pensarse que en estos supuestos está presente la ventaja de ser una intervención sobre uno mismo; es la propia persona la que decide sobre su cuerpo y sería una suerte de ejercicio de la autonomía individual ${ }^{48}$.

Sin embargo esto no disminuye la complejidad del asunto, ni la posibilidad de discriminación.

$Y$ es que, como es sabido, uno de los grandes problemas éticos de estas tecnologías tiene que ver con la garantía de una igualdad de oportunidades para su disfrute $y$, por ende, con la posibilidad de

\footnotetext{
${ }^{46}$ Agar, N., Liberal Eugenics. In Defense of Human Enhancement, Blackwell, Oxford 2004 , p. 155.

47 Lema, C., "Intervenciones biomédicas de mejora, mejoras objetivas y mejoras discriminatorias: ¿De la Eugenia al darwinismo social?", cit., p. 388.

48 Vid. esta argumentación en relación con el uso de fármacos en Caplan, A.,"Is Better Best? A Noted Ethicist Argues in Favor of Brain Enhancement", en Scientific American 289, 2003, pp. 104.
} 
aumentar la brecha entre ricos y pobres (ya sean personas o países) ${ }^{49}$.

No cabe duda de que si en el ejemplo que puse anteriormente relativo al chip de idioma, añadiera la información de que es más fácil encontrar trabajo si comprendes más lenguas, y además, incorporara datos sobre el coste de cada uno de los chips, la discusión se haría incluso más compleja.

En este sentido, la filósofa estadounidense Martha Nussbaum, comienza su trabajo "Genética y Justicia: Tratar la enfermedad, respetar la diferencia", con el siguiente supuesto tomado del libro de Buchanan, Brock, Daniels y Wikler, Genética y Justicia: "Katherine y Bill concurren al mismo puesto directivo en una gran empresa. La solicitud de Katherine incluye un certificado de mejoramiento genético de Opti-Gene, el cual establece que su titular ha adquirido un conjunto de servicios genéticos destinados a aumentar la memoria y potenciar el sistema inmunológico. Bill, que no se puede permitir un mejoramiento genético, objeta que un contrato basado en la mejora genética supone una violación de la igualdad de oportunidades: el trabajo debería adjudicarse en función del mérito. Katherine replica que adjudicar el trabajo en función del mérito significa que el puesto corresponde al mejor candidato, y ella es la mejor candidata, de manera que ¿dónde está el problema?"50.

Como señala M. Nussbaum, la disputa entre Katherine y Bill "no dista mucho de los problemas relativos a la igualdad de oportunidades que durante largo tiempo hemos estado debatiendo". Así, algunos podrán decir que Katherine se ha visto favorecida al pertenecer a una familia de clase media, teniendo acceso por ello a servicios de salud y educación, o por heredar de los genes de sus padres buenas cualidades; pero otros negarán que esto debe ser tenido en cuenta... Sin embargo, y de nuevo en palabra de Nussbaum, la "controversia entre Katherine y Bill también plantea algunas cuestiones que no se pueden abordar a partir de las teorías éticas actuales. Todas las teorías éticas actuales establecen algún tipo de distinción entre el reino de la naturaleza o del azar y el reino de la justicia. Aunque no queda claro dónde está la línea divisoria en cada caso particular, parece como si nuestro sentido global de la vida dependiera de la existencia de dicha distinción. Algunas cosas que nos van mal son sólo tragedias, se encuentran más allá del control humano; otras se podrían haber evitado o controlado con una mejor planificación social $y$, de este modo, son propias del reino de la

\footnotetext{
49 No voy a referirme aquí a otro gran problema relacionado con la discriminación y las nuevas tecnologías, que se proyecta en los ciborgs, y que tiene que ver con sesgos y discriminaciones de los algoritmos. Vid. O'Neil, C., Armas de destrucción matemática. Cómo el Big Data aumenta la desigualdad y amenaza la democracia, Capitan Swing, Madrid 2017, pp. 131 y ss.

50 Nussbaum, M., "Genética y Justicia: Tratar la enfermedad, respetar la diferencia", trad. de R. Orsi, Isegoria, n. 27, 2002, p. 6.
} 
justicia social. La naturaleza no parece que vaya a desaparecer por ahora y, en cualquier caso, a todos nos aguarda un triste final. Pero lo que la historia de Katherine y Bill nos revela es que vivimos en un tiempo caracterizado por «la colonización de lo natural por lo justo». Muchas cosas que siempre se han visto como accidentes inmutables ahora parece que las podemos cambiar, y que tenemos incluso la obligación de cambiarlas" 51.

En el caso de los ciborgs, el que la intervención sea sobre uno mismo, y fruto de la libre elección de la persona, no tiene nada que ver con el hecho de que la misma pueda producir una ventaja social.

Esta situación fue bien descrita por Lee Silver, profesor de biología molecular en Princeton, en su obra publicada en 1998, Vuelta al Edén ${ }^{52}$. En ella, Silver presenta una situación futura caracterizada por la aparición de la reprogenética (unión de la medicina reproductiva y la genética), a través de la cual es posible diseñar las características biológicas de los descendientes. Silver señala que, a lo largo del tiempo, la sociedad que describe estará dividida entre "naturales" y "genricos"... Pues bien, en un mundo como el que describe Silver, parece fácil predecir a qué grupo pertenecerán las personas en situación de vulnerabilidad y cómo será la satisfacción de sus derechos...

Obviamente, la objeción de la discriminación, sirve para todos aquellos que defiendan una teoría de la justicia igualitaria, pero no es relevante para aquellos que no lo hagan ${ }^{53}$. Por otro lado, se ha argumentado que en una sociedad con personas mejoradas existirían más oportunidades de redistribuir fruto de la actuación de éstas, y menos personas a las que atender ${ }^{54}$

En todo caso, y más allá de la cuestión de la discriminación, puede haber intervenciones que pongan en peligro el desarrollo moral de la personalidad. Para muchos, este desarrollo implica la realización

51 Nussbaum, M., "Genética y Justicia: Tratar la enfermedad, respetar la diferencia", cit., pp. 6 y 7.

52 Silvers, L., Vuelta al edén. Más allá de la clonación en un mundo feliz, Taurus, Madrid 1998.

53 Por ejemplo, J. Savulescu defiende prácticas genéticas de mejora con independencia de que a través de ellas se incremente la desigualdad social. Vid. Savulescu, J., ¿Decisiones peligrosas?: Una bioética desafiante, Tecnos, Madrid, 2012 , p. 45. En general, sobre la cuestión de la identidad en la sociedad digital vid. Piñar, J.L., "Identidad y persona en la era digital", en AA.VV., Sociedad Digital y Derecho, BOE, Madrid 2018, pp. 95 y ss. También y en lo referente a los ciborgs, Pérez-Luño, A.E., "Las generaciones de derechos humanos ante el desafío posthumanista", en AA.VV., Sociedad Digital y Derecho, cit., p. 151.

54 Vid. Harris, J., Enchancing Evolution. The Ethical Case for Making Better People, cit., p. 120. Desde planteamientos feministas S. Firestone ha defendido la reproducción artificial como medio de liberalización de la mujer. Vid. Firestone, S., La dialéctica feminista: en defensa de la revolución feminista, Kairós, Barcelona 1974. Sobre el tema vid. Puigpelat, F., "Feminismo y técnicas de reproducción asistida", en Marcos del Cano, A.M. (coord.), Bioética y derechos humanos, UNED, Madrid 2011, pp. 105 y ss. 
de esfuerzos y sacrificios que pueden desaparecer con las tecnologías de mejora. Con estas tecnologías puede reducirse sensiblemente nuestro sentido de autonomía y responsabilidad ${ }^{55}$ y por tanto nuestra propia agencia moral ${ }^{56}$.

En un trabajo sobre la necesidad de una reflexión ética en relación con el uso de las nuevas tecnologías, un grupo de científicos encabezado por Rafael Yuste, se han referido a como las neurotecnologías pueden llegar a alterar claramente el sentido de la identidad y agencia de las personas, y sacudir las suposiciones básicas sobre la naturaleza del yo57.

En este sentido, J. Habermas, desde la idea de dignidad humana, entendida como aquello con los que nos reconocemos como seres de una misma especie, ha defendido que toda intervención que altere aquello que permite ese reconocimiento, no estaría justificada ${ }^{58}$.

Ciertamente resulta difícil establecer cuales son los rasgos que nos identifican y singularizan, y defender que se mantienen de manera constante a lo largo de la historia de la humanidad. Así, algunos autores defienden que las personas a lo largo de su vida sufren cambios radicales, esto es, se ven afectadas por mejoras radicales naturales que no afectan a su identidad personal ni se cuestionan en términos de especie. Y si esto es así, ¿por qué lo van a hacer las mejoras radicales artificiales? ${ }^{59}$.

Como puede observarse, volvemos al principio, esto es, a la evaluación que toma como referente la idea de normalidad... Hace unos años se publicó un estudio que establecía como límite natural de la vida de un ser humano en 115 años (aún así, se aludía a casos excepcionales en los que se había llegado a los 122 años). Imaginemos que fuera posible ampliar la vida humana de una persona. Pues bien, lo que se vendría a defender desde las posiciones que acabamos de ver es que toda intervención que buscara alcanzar los 115 años no tendría que plantear problemas más allá de los

\footnotetext{
55 Vid. Ach, J.S., "Improving human performance?", en J. S. Ach, J.S. y Luttenberg, B. (eds.), Nano- biotechnology, nanomedicine and human enhancement, Berlín 2008.

56 Como vemos, aquí también está presente la eterna cuestión del paternalismo y su justificación. Al respecto vid. Ramiro, M.A., "A vueltas con el paternalismo", Derechos y Libertades, n. 15, 2006, pp. 221 y ss.: Campoy, I., La fundamentación de los derechos de los niños, Dykinson, Madrid 2006, pp. 984 y ss.

57 Vid. Yuste, R., y otros, "Four ethical priorities for neurotechnologies and $\mathrm{AI}^{\prime \prime}$ en Nature, Nov 8, pp. 159 y ss.

58 Vid. Habermas, J., El futuro de la naturaleza humana. ¿Hacia una eugenesia liberal?, Paidós, Barcelona 2010, p. 50.

59 Bostrom, N. y Ord, T., "The Reversal Test: Eliminating Status Quo Bias in Applied Ethics", en Ethics 116, 2006, pp. 656 y ss.
} 
derivados del aumento de la población ${ }^{60}$. La mejora de la población dentro de los límites naturales salvaría los problemas planteados por autores como J. Habermas y no sería una mejora radical en los términos de N. Agar ${ }^{61}$. Sin embargo, en este ejemplo vuelven a aparecer los problemas apuntados de la relatividad de la mejora, de la discriminación o de la afectación a terceros.

Como acabamos de ver, existen muchas cuestiones éticas abiertas. $Y$ es claro que no son las únicas ${ }^{62}$ y que no solo nos plantean "desventajas" éticas sino también "oportunidades". Se trata de cuestiones difíciles de resolver pero no por ello irresolubles. Es posible, y necesario, establecer líneas claras que nos permitan establecer incentivos y límites. Dejar este problema en manos del libre juego del mercado o del deseo humano no parece ser la mejor opción.

Resulta necesario llevar a cabo una reflexión interdisciplinar que nos permita alcanzar acuerdos sobre cómo regular estas prácticas. Pero todo ello debe hacerse desde unos referentes, desde un marco. $Y$ en este punto, si nos preguntamos cual es el marco general que en los últimos siglos ha construido la humanidad para hacer frente a los problemas que nos acompañan, la respuesta es clara: los derechos humanos.

\section{3.- LOS DERECHOS HUMANOS}

Hace cuatro años publicaba Una mirada a la robótica desde los derechos humanos, defendiendo precisamente esa idea al referirme al impacto de las tecnologías emergentes ${ }^{63}$. La humanidad ha tenido siempre que reaccionar ante los cambios tecnológicos. $\mathrm{Y}$ esto ha sido mucho más urgente en momentos de cambios revolucionarios. Pero siempre hemos alcanzado un nuevo equilibrio. Ahora bien, hoy estamos mejor posicionados que nuestros antepasados.

Hace más de 70 años, en diciembre de 1948, se aprobó la Declaración Universal de los Derechos Humanos. Bobbio, el conocido filósofo italiano, dijo hace tiempo que la Declaración debía ser el referente de la regulación internacional y que su aprobación se manifestaba la existencia de un consenso internacional sobre su

60 Vid. este ejemplo en Parra Sáez, J., "La mejora humana. Más allá de la radicalidad", Actas II Congreso de la Red Española de Filosofía, vol. II, 2017, pp. 57 y ss.

${ }^{61}$ Agar, Nicholas, Truly human enhancement: A philosophical defense of limits, MIT Press, 2014, Prefacio, xi.

62 En Una mirada a la robótica desde los derechos humanos he señalado además otros problemas como el impacto sobre nuestra identidad en términos de dependencia o de aislamiento social, el impacto sobre la diversidad cultural y biológica, el impacto sobre el medio ambiente, sobre la idea de cooperación, sobre la solidaridad...

63 Cit., pp. 54 y ss. 
significado y alcance ${ }^{64}$. Ciertamente, la historia nos ha demostrado la debilidad de ese consenso y la importancia de seguir justificando la virtualidad de los derechos y su capacidad para responder a los viejos y nuevos problemas de la humanidad.

La idea de que los derechos humanos deben ser el referente desde el que enfrentarse a los desafíos de las nuevas tecnologías está calando65. Así, el Instituto de Ingeniería Eléctrica y Electrónica (IEEE), asociación mundial de ingenieros, por ejemplo lleva tiempo discutiendo sobre beneficios y problemas de las nuevas tecnologías tomando como referencia los derechos humanos. En el campo de la bioética y en el de las tecnologías de la información y comunicación se ha defendido esta idea ${ }^{66}$.

En 2017, en un artículo publicado en Nature, un grupo de científicos abogaba por la creación de una convención internacional para definir acciones prohibidas relacionadas con la neurotecnología tomando como referencia los derechos ${ }^{67}$. Y algo parecido está ocurriendo en el campo de la Inteligencia Artificial.

Así, en 2017, el Instituto Rathenau, comisionado por la Asamblea Parlamentaria del Consejo de Europa (PACE), emitió el informe "Derechos Humanos en la era robótica", en la que, entre otras cosas recomendaba la creación de una Convención para la defensa de los derechos humanos en la era de la robótica.

$Y$ es que una sola aplicación de Inteligencia Artificial puede impactar una gran cantidad de derechos civiles, políticos, económicos, sociales y culturales, con impactos positivos y negativos simultáneos en el mismo derecho para diferentes personas ${ }^{68}$.

Un estudio publicado por el Berkman Klein Center de la Universidad de Harvard en septiembre de 2018, sobre inteligencia artificial y derechos humanos ha destacado este carácter complejo examinando su proyección es aspectos muy diferentes como pueden ser los procesos de préstamo, la salud, la contratación, la

64 Vid. Bobbio, N., "Sobre el fundamento de los derechos del hombre", en El tiempo de los derechos, Sistema, Madrid 1991, p. 61.

65 En el campo de la bioética esto es así desde hace tiempo. Vid. Morente Parra, V., Nuevos retos biotecnológicos para los derechos fundamentales, Comares, Granada 2014; Marín Castán, M.L., "En torno a la dignidad humana como fundamento de la Declaración Universal sobre Bioética y Derechos Humanos de la UNESCO", en Revista de Bioética y Derecho, núm. 31, 2014, pp. 17-37.

66 Vid. por ejemplo en España, en el campo de la bioética Marcos del Cano, A.M., "La Bioética y el Bioderecho desde los Derechos Humanos", en Marcos del Cano, A.M., Bioética y derechos humanos, cit., p. 43; y en el campo de las tecnologías de la información y la comunicación, Garriga, A., Nuevos retos para la protección de datos personales, Dykinson, Madrid 2015, pp. 57 y ss.

67 Vid. Yuste, R., y otros, "Four ethical priorities for neurotechnologies and AI", cit., pp. 159 y ss.

68 Sobre las ventajas y los riesgos de la inteligencia artificial desde el punto de vista de los derechos, vid. Martínez, R., "Inteligencia artificial, Derecho y derechos fundamentales", en AA.VV., Sociedad Digital y Derecho, BOE, Madrid 2018, pp. 259 y ss. 
información...69. Pero además, lo que este estudio pone de manifiesto es que desde el campo de la inteligencia artificial se está trabajando para evitar los problemas que su uso pueden producir en el ámbito de los derechos humanos.

En el informe de 2018 del Ethics Advisory Group del Supervisor Europeo de Protección de Datos, titulado "Towards a digital ethics", se afirma como una de las grandes conclusiones que "la vida digital tendrá que ser compatible con la naturaleza inviolable de la dignidad humana"70.

En mayo de 2018, después de discusiones y entrevistas con docenas de expertos en inteligencia artificial, en relaciones entre empresas y derechos humanos, y en otros temas variados, se aprobó la "Declaración de Toronto" (firmada por Amnistía Internacional, Access Now, el Observatorio de Derechos Humanos y la Fundación Wikipedia, entre otros) ${ }^{71}$.

La Declaración tiene tres secciones principales. En la primera, se establece el deber de los Estados de prevenir la discriminación en el diseño o la implementación de los sistemas de aprendizaje automático en contextos públicos o mediante asociaciones públicoprivadas. En la sección se incluyen principios para identificar riesgos en el uso de sistemas de aprendizaje automático, para garantizar la transparencia y la rendición de cuentas, para hacer cumplir la supervisión y para promover la igualdad. En la segunda sección se describe las responsabilidades de los actores privados en el contexto del desarrollo y la implementación de sistemas de IA. Estas responsabilidades incluyen detectar posibles resultados discriminatorios a través de la identificación y evaluación de riesgos, tomar medidas efectivas para prevenir y mitigar la discriminación y ser transparentes. Y en la tercera sección se afirma el derecho a un recurso efectivo y a llamar a cuentas a los responsables de las violaciones.

La Declaración de Toronto busca proteger los derechos de todos los individuos y grupos y promover la diversidad y la inclusión. Subraya que la inclusión, la diversidad y la equidad son componentes clave para garantizar que los sistemas de aprendizaje automático no creen ni perpetúen la discriminación, en particular contra los grupos marginados.

69 Raso, F.A., Hilligoss, H., Krishnamurthy, V., Christopher Bavitz, Ch., Kim, L., Artificial Intelligence \& Human Rights: Opportunities \& Risks, The Berkman Klein Center for Internet \& Society, Universidad de Harvard, septiembre de 2018. Disponible en https://cyber.harvard.edu/sites/default/files/2018-09/201809 AIHumanRightsSmall.pdf? (consultado el 14 de octubre de 2018)

70 Disponible en https://edps.europa.eu/sites/edp/files/publication/18-0125 eag report en.pdf, consultado el 21 de diciembre de 2018.

71 Disponible en https://www.accessnow.org/cms/assets/uploads/2018/08/TheToronto-Declaration ENG 08-2018.pdf (consultada el 3 de noviembre de 2018). 
Sundar Pichai, CEO de Google, firmó, el 7 de junio de 2018, el documento "IA y Google: nuestros principios" no desarrollar tecnologías cuyo propósito contraviene los principios ampliamente aceptados del derecho internacional y los derechos humanos 72 .

La Comisión Europea mantuvo, el 18 de junio de 2018, una reunión de alto nivel con 12 representantes de organizaciones filosóficas y no confesionales de toda Europa, en la que se abordaron las repercusiones de la IA sobre los derechos fundamentales, y sus consecuencias para la inclusión social y el trabajo.

El 23 de octubre de 2018, la organización The Public Voice, aprobó las "Directrices Universales para la Inteligencia Artificial", documento respaldado por 50 organizaciones científicas y más de 200 expertos de todo el mundo, que tiene como objetivo maximizar los beneficios de la IA, minimizar el riesgo y garantizar la protección de los derechos humanos ${ }^{73}$.

Es posible citar en este sentido también la "Declaración sobre ética y protección de datos en la Inteligencia Artificial" acordada por la Conferencia Internacional de instituciones dedicadas a la protección de datos y la privacidad (ICDPPC), de 25 de octubre de $2018^{74}$. En esta Declaración se reconoce que los sistemas de inteligencia artificial pueden traer beneficios significativos para los usuarios y la sociedad, pero al mismo tiempo pueden cuestionar el respeto de los derechos a la privacidad y la protección de datos y que este desarrollo debe complementarse con consideraciones éticas y de derechos humanos.

Así, la Declaración afirma que cualquier creación, desarrollo y uso de sistemas de inteligencia artificial debe respetar plenamente los derechos humanos, en particular los derechos a la protección de datos personales y privacidad, así como la dignidad humana y la no discriminación.

Los derechos humanos deben ser así el marco desde el que afrontar los retos éticos de las nuevas tecnologías ${ }^{75}$. Y los responsables de que esto sea así son, en primer lugar, los Estados y las organizaciones internacionales como Naciones Unidas. Pero esa responsabilidad nos compete a todos $\mathrm{y}$, como no, a las empresas e industrias.

En este punto se pone de manifiesto uno de los grandes problemas que afectan a la satisfacción de los derechos. El que hasta

72 Disponible en https://www.blog.google/technology/ai/ai-principles/ (consultado el 18 de diciembre de 2018).

73 Disponible en

https://thepublicvoice.org/ai-universal-guidelines/ (consultado el 15 de diciembre de 2018).

74 Disponible en

https://icdppc.org/wp-content/uploads/2018/10/20180922 ICDPPC-40th AIDeclaration ADOPTED.pdf (consultada el 3 de noviembre de 2018).

75 Vid. recientemente Llano Alonso, F.H., Homo Excelsior. Los límites ético-jurídicos del transhumanismo, cit., pp. 171 y ss. 
hace poco no se haya hecho referencia a los derechos humanos como el marco desde el que abordar los avances de las nuevas tecnologías, no es otra cosa que una manifestación de las dificultades con las que se encuentran los derechos en el ámbito de las relaciones privadas. El de los derechos en las relaciones entre particulares es un problema que considerábamos resuelto desde un punto de vista teórico pero que no lo está. Y no lo está claramente en el campo de la práctica y me temo que tampoco en el de la teoría. Por eso tenemos que insistir en ello: los derechos humanos no solo limitan la actuación de los poderes públicos, sino también la de los ciudadanos y la de las empresas y las industrias ${ }^{76}$.

En todo caso, en el campo de las empresas e industrias, es importante que seamos conscientes de la existencia de los "Principios Rectores de la ONU sobre las Empresas y los Derechos Humanos" que el Consejo de Derechos Humanos de Naciones Unidas hizo suyos en junio de $2011^{77}$.

En virtud de esta declaración los Estados deben proteger contra las violaciones de los derechos humanos cometidas en su territorio y/o su jurisdicción por terceros, incluidas las empresas; y en este sentido, deben adoptar las medidas apropiadas para asegurar la eficacia de los mecanismos judiciales nacionales cuando aborden las violaciones de derechos humanos relacionadas con empresas, en particular considerando la forma de limitar los obstáculos legales, prácticos y de otros tipos que puedan conducir a una denegación del acceso a los mecanismos de reparación.

En la declaración, se afirma que las empresas deben respetar los derechos humanos internacionalmente reconocidos - que abarcan, como mínimo, los derechos enunciados en la Carta Internacional de Derechos Humanos y los principios relativos a los derechos fundamentales establecidos en la Declaración de la Organización Internacional del Trabajo relativa a los principios y derechos fundamentales en el trabajo. Esta obligación de respetar los derechos humanos se aplica a todas las empresas independientemente de su tamaño, sector, contexto operacional, propietario y estructura. Igualmente, en este ámbito se proclama que las empresas deben proceder con la debida diligencia en materia de derechos humanos, y que este proceso debe incluir una evaluación del impacto real y potencial de las actividades sobre los derechos humanos, la integración de las conclusiones, y la actuación al respecto; el seguimiento de las respuestas y la comunicación de la forma en que se hace frente a las consecuencias negativas. Además se afirma que si se determina que han provocado o contribuido a

\footnotetext{
76 Vid. De Asís, R., Las paradojas de los derechos fundamentales como límites al Poder, Dykinson, Madrid 2000.

77 Pueden consultarse en

https://www.ohchr.org/documents/publications/guidingprinciplesbusinesshr sp.pdf
} 
provocar consecuencias negativas deben repararlas o contribuir a su reparación por medios legítimos.

Por otro lado, el discurso de los derechos humanos se ha proyectado directamente en la reflexión sobre los derechos de los propios ciborgs. En este punto es de destacar la Declaración sobre los derechos de los ciborgs ${ }^{78}$ elaborada por Aral Balkan, quien se define como un activista por los derechos de los ciborgs. Pues bien, en dicha Declaración se afirma, entre otras cosas que: "Los seres humanos en la era digital utilizan las tecnologías digitales para ampliar sus mentes y, por lo tanto, su ser... La relación de un ser humano con la tecnología digital es la de un organismo con sus órganos. Los órganos digitales de un ser humano pueden residir tanto dentro como fuera... Los seres humanos en la era digital son ciborgs...". Y desde todo lo anterior de declara que: "Los artículos de La Declaración Universal de Derechos Humanos se aplican a la definición de seres humanos en la era digital tal como se define en esta Declaración Universal de los Derechos del Ciborg y protegen la integridad y la dignidad del yo cibernético".

De esta forma, los derechos humanos comienzan a ser el referente tanto para la "industria ciborg" como para los propios ciborgs...

Obviamente, tomar como referencia los derechos no va a servir para solucionar todos los problemas que nos plantean las nuevas tecnologías. Sin embargo si que puede servirnos para reorientar algunas de las discusiones al respecto. Así por ejemplo, si fuéramos capaces de conseguir aclarar cuando estamos ante terapia y cuando ante mejora, y cuando son aceptables estas prácticas desde el punto de vista ético, la aproximación basada en derechos llamaría la atención sobre la cuestión de las barreras sociales y la discriminación. Esto puede ser ilustrado acercándonos a los problemas que plantea la relación entre nuevas tecnologías y discapacidad desde el punto de vista de los derechos ${ }^{79}$.

Como es sabido, la discapacidad se describe por la conjunción de dos circunstancias: condición y situación. La Convención de Naciones Unidas sobre los Derechos de las Personas con Discapacidad, en su artículo 1 señala: "Las personas con discapacidad incluyen a aquellas que tengan deficiencias físicas, mentales, intelectuales o sensoriales a largo plazo que, al interactuar con diversas barreras, puedan impedir su participación plena y efectiva en la sociedad, en igualdad de condiciones con las demás". Pues bien, en muchos casos, entre los logros que suelen asociarse a las nuevas tecnologías está el conseguir reducir los efectos de las deficiencias o

78 Disponible en https://cyborgrights.eu/\#discourse-comments, consultada el 9 de enero de 2019.

79 Sobre el tema vid. Romañach, J., Bioética al otro lado del espejo, Diversitas, Santiago de Compostela, 2009. 
incluso, terminar con ellos. Sin embargo, algunos defensores de los derechos de las personas con discapacidad han criticado este uso de las nuevas tecnologías afirmando que la admisión de intervenciones de mejora (o incluso terapeúticas), aplicadas a alguien que posee alguna deficiencia, pueden ser contempladas como una minusvaloración de las personas que poseen esas deficiencias ${ }^{80}$.

También se ha criticado ese uso señalando como pueden ser prácticas que se enfrenten a la identidad y la diversidad humana. El siguiente texto de Martha Nussbaum es ilustrativo al respecto: "Y es que la vida humana siempre ha sostenido una lucha contra los límites de la naturaleza, y cada uno de los seres humanos es el resultado de dicha lucha. Es más, sabemos que muchas de las vidas humanas más valiosas y creativas son el resultado de una lucha especialmente difícil que alejó a ciertas personas de lo convencional y las convirtió en blanco del desprecio y el insulto de sus iguales. Cualquiera que haya sido un mal deportista, o que tenga un físico diferente de cierto estereotipo sexual, sabrá que en estos «defectos» está presente un sufrimiento genuino - de modo que un progenitor bondadoso haría bien en realizar alteraciones genéticas para prevenirlos, dando lugar así a una nación de mujeres con grandes pechos y hombres musculosos. Pero ¿no debería cambiar más bien la cultura que la naturaleza? ¿Y de dónde, en un mundo de estereotipos sexuales genéticamente modelados según el patrón de una cultura recalcitrante, surgirán los artistas e intelectuales? Si todos los niños pueden ser creados conforme a cualesquiera que sean las normas

80 Así, se afirma que "la lucha contra la existencia de la diversidad funcional es, desde mi perspectiva, una lucha contra la esencia de la humanidad. Esto implica que todo avance científico y tecnológico será insuficiente si aspira a la erradicación de la diversidad funcional, porque el ser humano seguirá siendo como es, diverso en su funcionalidad a lo largo del tiempo, y por lo tanto diverso en su capacidad a lo largo de su vida". Romañach, J., "Cyborgs, mentes, máquinas y diversidad funcional: Una visión desde el otro lado del espejo", 2012, disponible en http://www.diversocracia.org/docs/Cyborgs mentes maquinas y diversidad\%20fu ncional def.doc. Un ejemplo paradigmático en el tratamiento de esta cuestión lo constituye la Sentencia del Tribunal Supremo de los Estados Unidos del caso Buck v. Bell de 1927 (disponible en http://caselaw.Ip.findlaw.com/cgibin/getcase. pl court $=$ us\&vol=274\&invol $=200$, consultado el 20 de octubre de 2013). El caso trata sobre una persona con discapacidad (hija de una persona con discapacidad y que había tenido otra hija con discapacidad) que es internada en una institución y que iba a ser esterilizada en virtud de una Ley. La sentencia confirma la validez de la Ley. Y quien redacta la conclusión es Oliver Wendell Holmes, quien afirma que si el bienestar público puede reclamar en ocasiones las vidas de los mejores ciudadanos con más razón podrá justificar unos sacrificios menores (la esterilización) a aquellos que constituyen una carga para el país (las personas con discapacidad). Así, se dice que para todos es mejor impedir la perpetuación de los no aptos. Y termina afirmando: "Tres generaciones de imbéciles son suficientes". 
dominantes en la sociedad, ¿no se empobrecerá por ello la vida humana?"81

Por último, se ha afirmado que en este ámbito, debería ser prioritario la lucha contra las barreras y no la lucha contra las llamadas deficiencias. Me he referido antes al ejemplo que aludía Agar en relación con la posibilidad de cambiar el color de la piel de los hijos, destacando como esta práctica solo tendría sentido como medida de protección del sol ante la disminución de la capa de ozono y nunca como intervención para evitar la discriminación en una sociedad racista ${ }^{82}$. Pues bien, desde este argumento se señalaría que la estrategia adecuada ante este ejemplo es la de detener la desaparición de la capa de ozono y evitar el racismo. En nuestro ejemplo de la prótesis de la pierna que se origina por las barreras que se encuentra una persona en silla de ruedas, la cuestión es ¿por qué no llevar a cabo una estrategia que busque la desaparición de las barreras? Así, en el campo de la tecnología, esto podría traducirse en el sentido de dar prioridad a la accesibilidad ${ }^{83}$

De alguna manera, el enfoque de derechos va a priorizar la eliminación de barreras sobre la lucha con la desaparición de las deficiencias, al igual que va a priorizar esta lucha frente a los programas de mejora.

En todo caso, conviene advertir que el enfoque de derechos no está reñido con el avance tecnológico. Como he señalado en otro lugar ${ }^{84}$, el discurso de los derechos humanos debe abrirse al desarrollo tecnológico y científico, y con ello: (i) no puede cerrar la puerta a toda intervención de mejora; (ii) debe ser capaz de combinar el antropocentrismo con visiones ecocéntrica y biocéntricas, que implique el respeto a la vida y al ser en su conjunto; (iii) debe atender a nuestra dimensión social y ser consciente de la importancia de las relaciones interpersonales para la consecución de una vida humana digna, configurando así una ética que tenga en cuenta al otro; (iv) debe prestar atención a las generaciones futuras poniendo especial énfasis en la responsabilidad y la previsión, en una responsabilidad orientada hacia el futuro que nos permita progresar con cautela, esto es, en una responsabilidad intergeneracional; (v) debe manejar una visión crítica con la idea de dignidad humana tal y como la hemos construido y concretado desde la modernidad y la ilustración, al estar basada en una concepción del ser humano y de la

\footnotetext{
81 Nussbaum, M., "Genética y Justicia: Tratar la enfermedad, respetar la diferencia", cit., pp. 15 y 16.

${ }^{82} \mathrm{Agar}$, N., Liberal Eugenics. In Defense of Human Enhancement, cit., p. 155.

83 Es recomendable en este punto la lectura del libro de Kalbag, L., Accesibility for Everyone, A Book Apart, New York 2017, y del manifiesto Ethical Design Manifesto disponible en https://2017.ind.ie/about/manifesto/, consultado el 8 de enero de 2019.

84 De Asís, R., "Robótica, Inteligencia Artificial y Derecho", en Revista de Privacidad y Derecho Digital, n. 10, 2018, pp. 65 y 66.
} 
sociedad de carácter universal y muy vinculada con la idea la perfección; (vi) debe ser igualmente crítica con la construcción tradicional de la igualdad, excesivamente ligada a la perspectiva formal, y partir de la diversidad como rasgo caracterizador de lo humano; (vii) debe encontrar un equilibro entre seguridad y libertad, y con ello, realizar un ejercicio de ponderación entre el principio de precaución y la libertad de investigación.

Para que el enfoque de los derechos consiga estar presente en la reflexión sobre las nuevas tecnologías no basta con que existan normas y declaraciones que así lo establezcan. Es necesario que los derechos estén presentes en el campo de la formación de todos aquellos implicados en las nuevas tecnologías. Es más, es necesario que los derechos estén presentes en el campo educativo en general.

$Y$ en este punto, tengo que reconocer la perplejidad que me produce observar como se potencia la incorporación en los colegios de la robótica (algo absolutamente necesario) y como, al mismo tiempo, disminuye e incluso se rechaza la educación en derechos humanos (que es algo igualmente necesario). 